\title{
Short-term Survival of Molar to Molar Transplantation in Adolescents
}

\author{
Seidu Adebayo Bello, ${ }^{1,2}$ John Adeoye, ${ }^{1}$ Nosa Ighile ${ }^{1}$ \\ ${ }^{1}$ State House Medical Centre, Aso-Rock, Abuja, Nigeria \\ ${ }^{2}$ QH Specialist Dental Clinics and Research Center, Gwarinpa, Abuja, Federal Capital Territory, Nigeria
}

Correspondence to: Dr. John Adeoye, State House Medical Centre, Aso-Rock, Abuja, Nigeria; email: jaadeoye@hku.hk

\begin{abstract}
Background: The first permanent molars are frequently affected by caries partly because they are the first teeth to erupt in the oral cavity and partly because of their susceptibility by virtue of their morphological and functional characteristics. Although extraction and replacement with osseointegrated implants may be acceptable in adult patients, these treatment options are not ideal in adolescents. Methods: This preliminary study was conducted in patients diagnosed with grossly carious and non-restorable molar teeth, and subsequently managed by molar to molar autotransplanting. Patients were recruited in a period of 2 years and had follow-up visits up to 3 years post-transplantation. Results: Eight cases of molar to molar autotransplanting were done in seven patients. Patients were within 15-17 years of age, with a mean age of $16.0+0.93$ years. The overall 3-year survival rate of autotransplanted mandibular molars in
\end{abstract}

this study is $75 \%$ with two cases of failure of treatment (25\%) occurring 6 months following the procedure. Conclusion: Molar to molar tooth autotransplanting in African adolescents may be considered for immediate teeth replacement in the management of teeth scheduled for dental extraction. More cases are required to validate the viability of the procedure.

Keywords: Adolescents, Tooth autotransplantation, Molar teeth, Survival rate

Ann Afr Surg. 2020; 17(1):11-15.

DOI: http://dx.doi.org/10.4314/aas.v17i1.4

Conflicts of Interest: None

Funding: None

(C) 2020 Author. This work is licensed under the Creative Commons Attribution 4.0 International License

\section{Introduction}

The first permanent molars, being the first in the tooth series to erupt in addition to their morphological and functional characteristics, have been shown to be the most affected by severe dental caries, pulpal and periapical pathologies $(1,2)$. In these cases, most dental surgeons may choose to perform an endodontic procedure with crown placement or to extract the tooth and rehabilitate afterwards with osseointegrated implants. However, in adolescents (12-17 years) osseointegrated implants may be challenging as they impede the normal growth of the alveolar process (3), and thus they do not fulfil the criterion for tooth rehabilitation in developmental adaptation in this age group $(4,5)$. A viable treatment alternative that satisfies this benchmark in addition to offering potential long-term survival in growing individuals is autogenous tooth transplanting.

Tooth autotransplanting is the transfer of embedded, impacted or erupted teeth from one site into extraction sockets or surgically prepared sites in the same individual (6). This method provides the potential to replace a missing tooth with a more natural option with the capacity for functional adaptation and preservation of the alveolar $(7,8)$. Earlier reports have highlighted special indications for this treatment option. These indications include maxillary incisor avulsion using developing mandibular second premolars as the donor, and loss of first permanent molars due to severe dental caries where the developing third molars may be considered a good source for autotransplanting $(2,9)$. Other indications include tooth agenesis, jaw reconstruction following tumor resection, ectopically positioned tooth and cervical root fracture $(10,11)$. However, treatment may be contraindicated in cases with poor self-motivation and unhealthy donor teeth and in recipient sites with insufficient multidimensional alveolar bone support (11).

The success rate of tooth autotransplanting according to literature is variable but impressive, ranging between $60 \%$ and $100 \%(6,7,12,13)$. Factors observed to affect the survival of autotransplanted teeth include patientrelated factors, donor tooth-related factors, recipient site factors and clinical factors. Better results were observed in younger patients, patients with good oral hygiene, and 
those free of major systemic and metabolic problems (13). Furthermore, it was observed that considering the healing of the dental pulp and continued development of the root, the ideal timing of transplantation is when the donor tooth root is $3 / 4$ to $4 / 5$ complete and placed in recipient sites with adequate bone height and width on the day of donor tooth removal, or within one month of extraction by an experienced clinician (13).

This study is borne out of the need to provide preliminary evidence for considering autogenous tooth transplanting as a viable treatment alternative for replacing missing teeth in Sub-Sahara African adolescents. Most studies on autotransplanting have been presented but emphases were more on canines and premolars. This present study presents our experience of molar to molar autotransplanting and evaluates the short-term success rate of this procedure.

\section{Patients and Methods}

This is a preliminary study of patients treated using molar to molar autotransplanting between June 2013 and September 2015. Patients in the age range 13-17 years with clinically and radiographically diagnosed grossly carious, non-restorable mandibular first or second molars were included in the study. Included subjects were free of major systemic or metabolic disease, or both, excellently motivated with good promise of complying with postoperative instructions and available for follow-up visits. Following clinical examination and radiographic investigations, adolescent subjects that were adjudged to benefit more from mesial drifting of distal tooth/teeth following the extraction procedure were excluded from the study. The donor teeth, which were all mandibular third molars, were selected as they were the only immature teeth in these adolescents and had a higher likelihood of impaction than any other teeth (14). These teeth were also selected according to their level of root development and apical closure. Teeth with root development of $1 / 2$ to $2 / 3$ with open apices were selected as this allowed for greater chance of success. Ethical approval was obtained from the Federal Capital Territory Health Research Ethics Committee and informed consent was obtained from subjects' parents before the procedure was carried out.

\section{Surgical procedure}

The procedure was carried out by an oral and maxillofacial surgeon together with two dental surgeons with prior understanding of the surgical procedure of autogenous tooth transplanting (Fig. 1).

Radiographs were taken of periapical and panoramic views. Antibiotics and analgesics were prescribed to subjects with existing periodontal or periapical pathology. Armamentarium included dental needle and syringe, lignocaine, mouth mirror, dental explorer, periosteal elevators, surgical drill, curettes, Coupland elevators, periotomes, lower molar extraction forceps, sutures, needle-holding forceps, surgical blades and Bard-Parker surgical blade handle.

Most patients had a single tooth transplated, thus the procedure was done under local anesthesia (2\% lignocaine in 1:80,000 adrenaline); one patient had bilateral autogenous tooth transplanting under general anesthesia. The procedure started after anesthesia was administered; next, a three-sided mucoperiosteal flap was raised at the site of the donor tooth and the tooth carefully luxated from its socket together with its complete root sheath. The donor tooth was placed back into the socket to keep it viable while the recipient bed was being prepared.

Preparation of the recipient bed included extracting the grossly carious tooth, curetting the root socket to remove any pathologic lining, and removing interradicular bone to create adequate space for the transplanted tooth. Bone was removed and curetted using a surgical drill, which resulted in increased mesio-distal width as well as the apico-coronal length. The donor tooth with its complete root sheath was then carefully placed in the prepared recipient bed. The operators ensured that the donor teeth were placed in infra-occlusion to prevent trauma from opposing teeth and allow for spontaneous eruption.

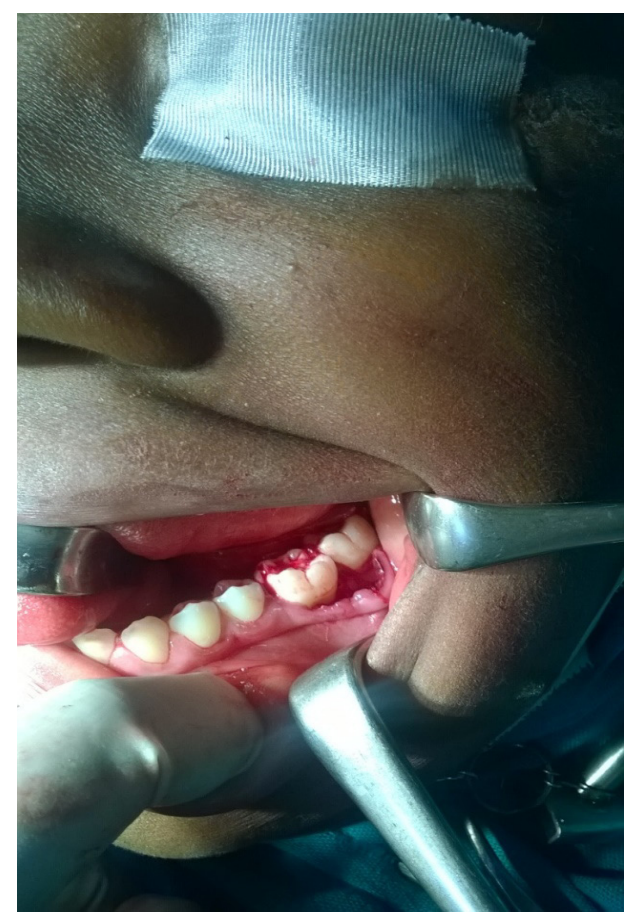

Figure 1. Immediate postoperative photograph of transplanted tooth 36 under local anaesthesia in a 14-year-old adolescent. 
Vicryl 2.0 sutures, composite materials or soft stainlesssteel wires were used to secure the tooth in the socket loosely and was left in situ for 48-72 hours. Follow-up was done 24 and 72 hours after transplanting and then a week later. At 1-week postoperative review, fluoride therapy of the transplant teeth was done as a measure to prevent development of dental caries following occlusal reduction. Subsequently, recall visits were done after 1, 3, 6 and 9 months, and 1, 2 and 3 years. Clinical and radiographic evaluation was done at each visit (Figs. 2 and 3). Data were collected periodically using designed data collection proforma.

Transplant tooth survival was determined based on strict clinical and radiographic criteria adapted from Cohen et al. (15), Pogrel et al. (16), Schwartz et al. (17) and Thomas et al. (18). Transplant tooth that did not satisfy any component of the clinical and radiographic criteria was deemed to have failed.

Clinical criteria were normal percussion sound, absence of tooth mobility, absence of pain or discomfort in subjects and evidence of good gingival healing with no indication of marginal attachment loss.

Radiographic criteria were crown to root ratio of $1: 1$, absence of external root resorption, absence of ankylosis, normal PDL space width around transplanted tooth, and absence of disturbance in root development.

Data were analysed using SPSS for Windows version 20. Data on radiographic and clinical findings obtained during follow-up periods were presented as tables and charts, and the relationships between clinical and radiographic parameters were identified. Pearson's chisquare test was used to compare differences between discrete variables. Kaplan-Meier survival analysis plots were used to graphically compare the percentage survival of the autotransplanted molars based on the stage of root development of the donor teeth. For all comparisons, $\mathrm{p}<0.05$ was inferred as the criterion for establishing

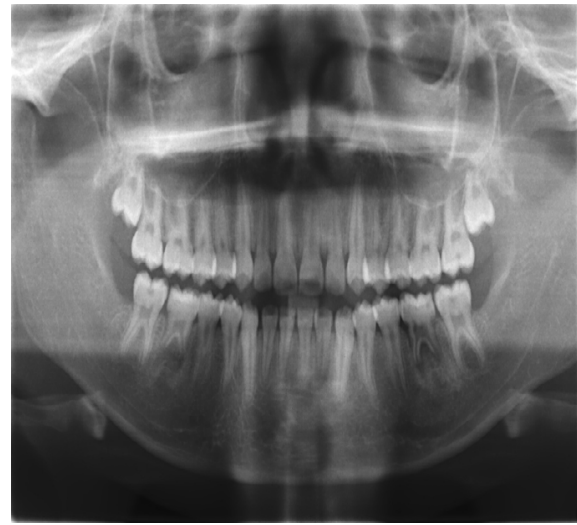

Figure 2. Immediate postoperative radiograph of transplanted tooth.

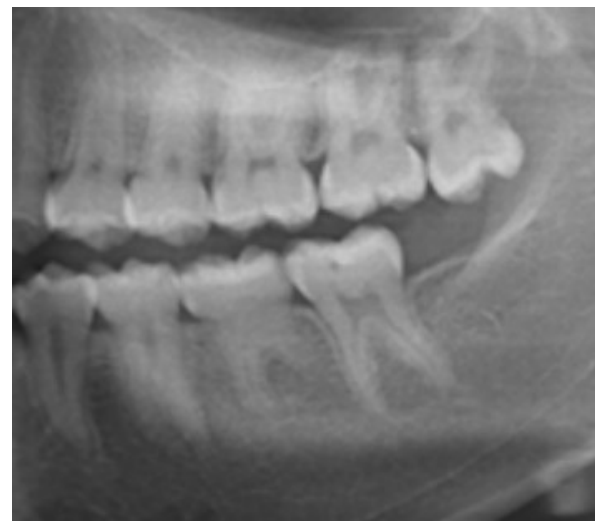

Figure 3. 3-year postoperative radiograph of the same patient in Figure 3.

\section{Discussion}

Autogenous tooth transplanting is a possible treatment option for replacing missing teeth and teeth earmarked for extraction especially in adolescents. However, most clinicians do not consider this option during treatment planning in cases involving this age group. This study demonstrated tooth autotransplanting of developing molar to molar teeth with a 3-year survival rate of $75 \%$. 
This rate is lower but comparable to the reports of Reich et al. (19) whose study of 44 autogenous maxillary and mandibular molar to molar transplanting yielded a success rate of $95.5 \%$.

This difference may be attributed to the wide disparity in the follow-up duration of each subject than to a more uniform and longer review rate in this study. Compared with premolar to premolar autogenous transplanting, the success rate in this study was lower though comparable to the reports of Czochrowska et al. (3), Tsukiboshi et al. (18) and Andreasen et al. (20) with survival rates of $79 \%, 82 \%$ and $90 \%$ respectively. Jonsson and Sigurdsson (19) reported a high cumulative survival rate of $92.5 \%$ involving premolar to premolar teeth autotransplanting. Two transplanted teeth were considered to have failed at the 6-month postoperative follow-up visit as they were grossly mobile, and were subsequently extracted. These were rehabilitated with osseointegrated single dental implants months later. Bello et al. (21) emphasized the importance of open apices to the survival rate of an autotransplanted tooth in a case of swinging replantation of an upper central incisor tooth, and as a build-up on that report all donor teeth in this study had open apices or incomplete roots. All transplanted teeth with roots more than half formed with open apices survived while $50 \%$ of transplant teeth with half-formed roots survived. This could mean that the chances of survival of a transplant tooth are higher if the donor tooth was extracted at a root development stage greater than half. Molar to molar autotransplanting also shows considerable promise as a rehabilitative option for prospective edentulous patients who satisfy the criteria, as its short-term survival rate compares satisfactorily

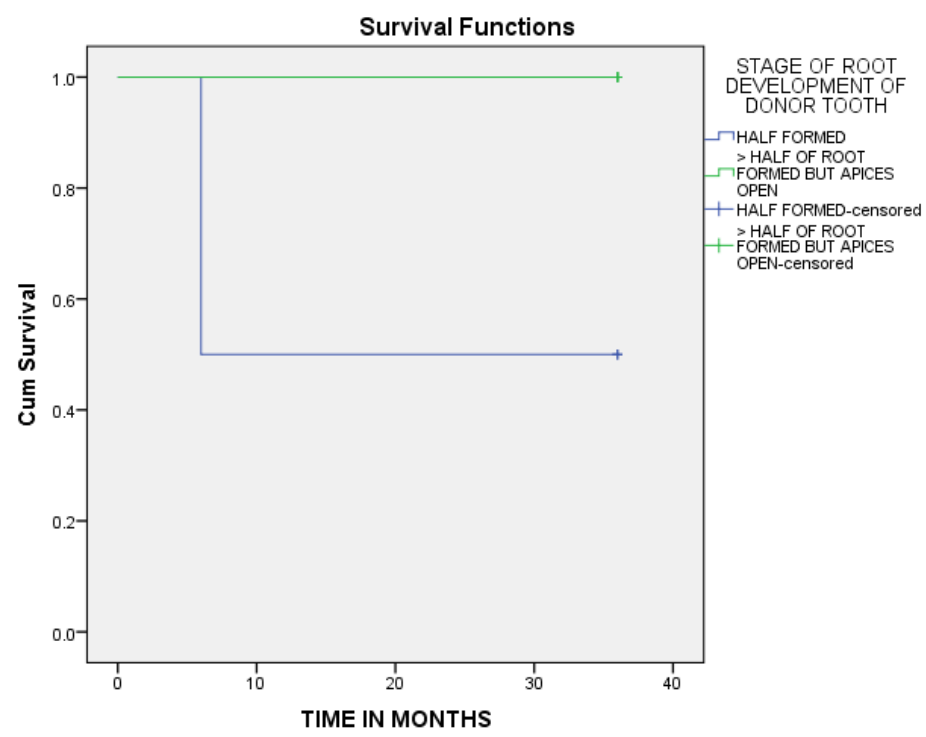

Figure 5. Kaplan Meier survival analysis plot based on the stage of root development of donor teeth. with that of other treatment options, such as resinbonded prostheses, in adolescents. Probster et al. (22) reported a 5-year survival rate of $76 \%$ from a study to determine the clinical performance of resin-bonded bridges, which is also similar to the report of this present study.

Several factors were observed to have contributed to the high survival rate of molar to molar autotransplanting after 3 years. First, all subjects and donor teeth were selected using strict criteria that ultimately favored the prognosis afterwards $(3,7,11,18-20)$. Second, in this study all donor teeth had the interproximal surfaces and crown cusps reduced to enable the donor teeth to be properly fitted into the recipient beds and to place the teeth in infraocclusion so as to prevent repeated contact and constant mobility following retention of the transplanted teeth (17). The recipient sites of all transplanted teeth were modified either by removing a portion of the inter-radicular bone or by curetting the sockets, or both, in cases with periapical infection. Finally, all transplantations in this study were performed by dental surgeons experienced in the surgical procedure and who adhered to a strict protocol (23).

The main drawback of molar to molar autotransplanting observed by the authors currently is the restricted number of teeth available to serve as donors in the procedure. If a donor tooth is deemed unfit for transplanting due either to morphologic features or iatrogenic error, the procedure will depend on harvesting the only other mandibular third molar available. This drawback was also reported by Reich et al. (19). It can be argued that the removal of the mandibular third molars may predispose to condylar fracture $(23,24)$ or cause distal drifting of the mandibular second molars. However, to the best of our knowledge, no report is available relating these drawbacks to this procedure either in this study or in the literature.

As this is a preliminary study with few subjects, it is limited by the sample size available for analysis, which influences the ability to make stronger conclusions as well as the external validity of our report.

\section{Conclusion}

Molar to molarautotransplanting maybe considered an option in the immediate rehabilitation of missing teeth in adolescents in Sub-Saharan Africa. However, to make concrete conclusions on the viability of the procedure in Africans, further studies, including more subjects reviewed over a longer duration, are necessary. 


\section{Acknowledgements}

We acknowledge the dental teams at State House Medical Centre and QH Specialist Dental Clinics and Research Centre for their contributions in the clinical aspects of this work.

\section{References}

1. Rafi A, Syed M, Zakirulla M, et al. Prevalence of first permanent molar caries among 7-10-year old school-going boys in Abha City, Saudi Arabia. J Int Oral Health. 2011; 3(5):29-34.

2. Patel A, Brennan J, Sandler P. Autotransplantation of an impacted third molar: An orthodontic case report. Dent Update. 2004; 31(10):596-8.

3. Czochrowska M, Stenvik A, Bjercke B, et al. Outcome of tooth transplantation: Survival and success rates 17-41 years post-treatment. Am J Ortho Dentofacial Orthop. 2002; 121(2):110-9.

4. Westwood R, Duncan J. Implants in adolescents: A literature review and case reports. Int J Oral Maxillofac Impl. 1996; 11(6):750-5.

5. Thilander B, Odman J, Grondahl K, et al. Aspects of osseointegrated implants inserted in the growing jaws: $\mathrm{A}$ biometric and radiographic study in the young pig. Eur J Orthod. 1992; 14:99-109.

6. Tanaka T, Deguchi T, Kageyama T, et al. Autotransplantation of 28 premolar donor teeth in 24 orthodontic patients. Angle Orthod. 2008; 78:12-9.

7. Slagsvold O, Bjercke B. Applicability of autotransplantation in cases of missing upper anterior teeth. Am J Orthod. 1978; 74:410-21.

8. CzochrowskaE,StenvikA,AlbumB, etal.Autotransplantation of premolars to replace maxillary incisors: A comparison with natural incisors. Am J Orthod Dentofacial Orthop. 2000; 118:592-600.

9. Zachrisson B. Planning esthetic treatment after avulsion of maxillary incisors. J Am Dent Assoc. 2008; 139:1484-90.

10. Marques-Ferreira M, Rabaça-Botelho M, Carvalho L et al. Autogenous tooth transplantation: Evaluation of pulp tissue regeneration. Med Oral Patol Oral Cir Bucal. 2011; 16:e984-9.
11. Josefsson E, Brattström V, Tegsjö U, et al. Treatment of lower second premolar agenesis by autotransplantation: Fouryear evaluation of 80 patients. Acta Odontol Scand. 1999; 57:111-5.

12. Tsukiboshi M. Autotransplantation of teeth: Requirements for predictable success. Dent Traumatol. 2002; 18:157-80.

13. Jonsson T, Sigurdsson T. Autotransplantation of premolars to premolar sites: A long-term follow-up. Am J Orthod Dentofacial Orthop. 2004; 125:668-75.

14. Carter K, Worthington S. Predictors of third molar impaction: A systemic review and meta-analysis. J Dent Res. 2016; 95(3):267-76.

15. Cohen A, Shen T, Pogrel M. Transplanting teeth successfully: Autografts and allografts that work. J Am Dent Assoc. 1995; 126:481-5.

16. Pogrel M. Evaluation of over 400 autogenous tooth transplants. J Oral Maxillofac Surg. 1987; 45:212-6.

17. Schwartz O, Bergmann P, Klausen B. Resorption of autotransplanted human teeth: A retrospective study of 291 transplantations over a period of 25 years. Int Endod J. 1985; 18:119-31.

18. Thomas S, Turner S, Sandy J. Autotransplantation of teeth: Is there a role? Br J Orthod. 1998; 25:275-82.

19. Reich P. Autogenous transplantation of maxillary and mandibular molars. J Oral Maxillofac Surg. 2008; 66:23147.

20. Andreasen J, Paulsen H, Yu Z, et al. A long-term study of 370 autotransplanted premolars parts I-IV. Eur J Orthod. 1990; 12:3-13.

21. Bello SA, Enebong DJ, Obi EV. Swinging replantation: A possible protocol for the management. J Contemp Dent Pract. 2017; 18(1):69-73.

22. Probster B, Henrich G. An 11-year follow-up study of resinbonded fixed partial dentures. Int J Prosthodont. 1997; 10:259-68.

23. Revanth K, Sinha R, Uppada U, et al. Mandibular third molar position influencing the condylar and angular fracture patterns. J Maxillofac Oral Surg. 2015; 14(4):956-61.

24. Armond A, Martins C, Glória J, et al. Influence of third molars in mandibular fractures. Part 2: Mandibular condyle-a meta-analysis. Int J Oral Maxillofac Surg. 2017; 46(6):7309. 